\title{
A CORRELATION ANALYSIS BETWEEN THE NUTRITIONAL STATUS AND PROGNOSIS OF COVID-19 PATIENTS
}

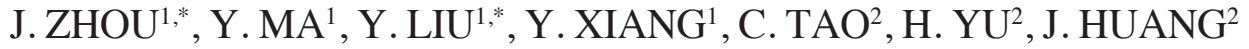 \\ 1. Department of Emergency, Hubei Provincial Hospital of Traditional Chinese Medicine,Wuhan, China; 2. Department of Radiology,Hubei Provincial Hospital of Traditional Chinese

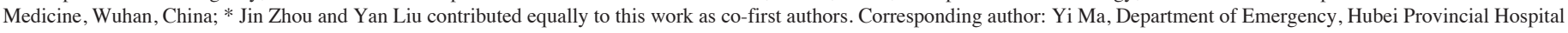 \\ of Traditional Chinese Medicine, Wuhan, China, 553776789@qq.com
}

\begin{abstract}
Purpose: The present study investigated the correlation between the nutritional status and prognosis of COVID-19 patients, and analyzed the epidemiological characteristics of COVID-19 patients with different nutritional status. Methods: 429 patients who were diagnosed positive for COVID-19 in Hubei Provincial Hospital of Traditional Chinese Medicine from December 2019 to March 2020 were selected and divided into different groups based on Controlling Nutritional Status (CONUT) score (0-4: the low CONUT score group; 5-12: the high CONUT score group). Multivariate logistic regression analysis was applied to investigate the effects of CONUT score on prognosis. Results: The total score of admission status of patients with higher CONUT score was higher than that of those with lower CONUT score $(\chi 2=7.152, \mathrm{P}=0.007)$. The number of adverse outcomes of female was higher than that of male $(\chi 2=10.253, \mathrm{P}=0.001)$. The number of adverse outcomes was higher for patients with smoking history $(\mathrm{P}=0.004)$ or hypertension $(\chi 2=11.240, \mathrm{P}=0.001)$ than those without. Also, the number of adverse outcomes was higher for older patients than younger ones $\left(\chi^{2}\right.$ $=15.681, \mathrm{P}<0.001)$. Patients with adverse outcomes had lower urine red blood cell count than patients without adverse outcomes $(\chi 2=5.029, \mathrm{P}=0.025)$. However, BMI, drinking history and diabetes did not show correlation with the prognosis of COVID-19 ( $\mathrm{P}>0.05$ ). Among patients $\geq 61$ years old, the risk of adverse outcomes in the high CONUT score group was 6.191 times that of the low CONUT score group (OR $=6.191,95 \%$ CI: 1.431 26.785).Among the non-diabetic patients, the risk of adverse outcomes in the high CONUT group was 11.678 times that of the low CONUT group (OR $=11.678,95 \%$ CI: $2.754-49.41)$.For the patients who had a total score of admission status $<6$, the risk of adverse outcomes in the high CONUT score group was 8.216 times that of the low CONUT score group (OR $=8.216,95 \%$ CI: 2.439-27.682). Conclusion: COVID-19 patients with good nutritional status showed a small chance to have adverse outcomes. Gender, age, hypertension, the number of urine red blood cell count and CONUT score affected the adverse outcomes of patients.
\end{abstract}

Key words: COVID-19, nutritional status, CONUT scor, prognosis.

The high infectivity of COVID-19 has led to a rapid increase in new cases and outbreaks since December 2019 (1, 2). A part of patients showed adverse outcomes from treatment, such as severe pneumonia, pulmonary edema, acute respiratory distress syndrome (ARDS) or even multiple organ failure. Recent studies have basically identified the correlation between the poor prognosis and outcome of COVID-19 patients with their own status (3). Based on the relationship between nutritional status and prognosis, the present study fully considered the nutritional status of patients in the acute phase of admission, and chose total cholesterol, total lymphocyte and serum protein count as CONUT score to reflect their nutritional status and immunological characteristics. Since the number of studies about the epidemiological characteristics of COVID19 is scarce, the present study aims to analyze the correlation between the nutritional status and prognosis of COVID-19 patients, and their epidemiological characteristics with different nutritional status.

\section{Methods and materials}

The present study used a single-center, retrospective analysis method. 489 patients who were diagnosed positive for COVID-19 in Hubei Provincial Hospital of Traditional Chinese
Medicine from December 2019 to March 2020 were recruited. However, 60 patients were excluded due to their missing score of CONUT, and 429 patients were finally included in the study. The diagnostic criteria was based on Diagnosis and Treatment Protocol for COVID-19 (Trial Version 7) (4). The study was approved by the Ethics Committee of Hubei Provincial Hospital of Traditional Chinese Medicine, and the data was from the cases in our hospital during hospitalization.

The average age of the included 429 patients was $58.29 \pm$ 15.89 (The oldest: 92; the youngest: 19). The number of male patients was $212(49.42 \%)$, and the number of female patients was $217(50.58 \%)$.

\section{Evaluation index}

The demographics, medical history, signs, symptoms and inpatient lab examinations of COVID-19 patients were collected. The major indicator of adverse outcomes was the all-cause death of COVID-19 patients. Secondary indicators of adverse outcomes included transferring to ICU due to aggravation, acute heart failure or acute respiratory failure (5). The CONUT score (the highest score: 12; the lowest score: 0 ) of each patient was evaluated based on his/her serum albumin, peripheral blood lymphocyte count, and cholesterol concentration (6-9). CONUT score 4 was set as the critical 


\section{THE JOURNAL OF NUTRITION, HEALTH \& AGING}

value, and patients were divided into the high-score and low-score group. Since serum albumin representing protein reserves, total cholesterol representing energy expenditure and lymphocyte count representing immune defense, a higher score refers to a worse nutritional status of patients. Thus, the CONUT score is used to predict the acute aggravation of surgical patients.

\section{Statistical analysis}

The data was analyzed by SPSS 20.0, and cases with complete clinical data were further selected for statistical analysis. Difference analysis was firstly done on the general data of groups with either high or low CONUT score, and then further difference analysis was done on the adverse outcomes (death, transferring to ICU, heart/respiratory failure). Data with normal distribution was expressed as mean \pm standard deviation (Student's t test). Data with skewed distribution was expressed as median and quartile $\mathrm{M}(\mathrm{Q} 25, \mathrm{Q} 75)$ (MannWhitney U test). Classified data was expressed as the number of cases and percentile N (\%) (Chi-square test or Fisher's exact test). Graded data was expressed as the number of cases and percentile N (\%) (Mann-Whitney U test) . Factors affecting the difference in clinical data between the high CONUT score group and the low CONUT score group were included in the logistic regression analysis of adverse outcomes as covariates in order to investigate the effects of CONUT score on adverse outcomes. $\mathrm{P}<0.05$ was considered as statistically significant.

\section{Results}

\section{Difference analysis of the high and low CONUT score groups}

Results showed that the proportion of diabetic patients in the high CONUT score group was higher than that in the low CONUT score group $\left(\chi^{2}=6.552, \mathrm{P}=0.010\right)$. The average age of the high CONUT score group was older than that of the low CONUT score group $\left(\chi^{2}=37.128, \mathrm{P}<0.001\right)$. The score of admission status of the high CONUT score group was also higher than that of the low CONUT score group $\left(\chi^{2}=7.152, \mathrm{P}\right.$ $=0.007)$. However, gender, BMI, smoking history did not show significant differences between the high and low CONUT score groups $(\mathrm{P}>0.05)$. More detailed information is shown in Table 1.

\section{Comparison of the differences in adverse outcomes in different groups}

Results showed that patients with adverse outcomes had a higher proportion of smoking history than patients without adverse outcomes $(P=0.004)$. Also, patients with adverse outcomes had a higher proportion of hypertension than patients without adverse outcomes $(\chi 2=11.240, P=0.001)$. The average age of patients with adverse outcomes was older than that of patients without adverse outcomes $(\chi 2=15.681, \mathrm{P}<$ $0.001)$. Patients with adverse outcomes also showed a lower urine red blood cell count than those without adverse outcomes $(\chi 2=5.029, \mathrm{P}=0.025)$. While, BMI, drinking history and diabetes did not show correlation with the adverse outcomes (P $>0.05)$. More detailed information is shown in Table 2.

\section{Multivariate regression analysis}

\section{Multivariate regression analysis of adverse outcomes}

Gender, age, smoking history, hypertension, coronary heart disease, urinary erythrocytes and other variates in Table 2 were included in the multivariate logistic regression (stepwise progression method). Results showed that gender, age, hypertension and urinary erythrocytes were the key factors affecting adverse outcomes. Detailed information is shown in Table 3.

Analysis of the influences of CONUT score on adverse outcomes

According to Table 3, gender, age, hypertension and urinary erythrocytes were the key variates affecting adverse outcomes. Diabetes, total score of admission status in Table 1 were included in the multivariate logistic regression of adverse outcomes as covariates. Results showed that gender, age, hypertension, urinary erythrocytes and CONUT score were the risky factors affecting adverse outcomes. More detailed information is presented in Table 4.

Analysis of the influences of CONUT score on adverse outcomes in different subgroups

The influences of age, diabetes and the total score of admission status on adverse outcomes under different nutritional status were further analyzed, and detailed information is shown in Table 5.

\section{Discussion}

The COVID-19 virus enters the cell through ACE2 receptor, which mainly locates in type 2 alveolar cells of the lung, upper and esophageal epithelial cells, cardiomyocytes and proximal renal tubules (10). The major features brought by COVID-19 are acute respiratory disease with diffuse alveolar hemorrhage and acute respiratory failure (11). However, the virus may enter the bloodstream and accumulate in other organs, such as heart, gastrointestinal tract and kidney, and cause further damage. Serum protein is an important factor of the three standards of CONUT, and also is a reliable index of systematic inflammation $(11,12)$. Pro-inflammatory cytokines, such as IL-6 and TNF- $\alpha$, and CRP can also decrease the concentration of serum albumin and regulate albumin synthesis by liver cells $(13,14)$. Low peripheral blood lymphocyte count is an indicator of insufficient host immune response (14). These three indicators can evaluate the general status of patients comprehensively, and thus we used CONUT score as an indicator to assess the nutritional status of patients. 
Table 1

Differences in the high and low CONUT score groups

\begin{tabular}{|c|c|c|c|c|c|}
\hline \multirow[t]{2}{*}{ Variates } & \multicolumn{2}{|c|}{ Groups } & \multirow[b]{2}{*}{$\begin{array}{c}\text { Cases of adverse } \\
\text { outcomes }\end{array}$} & \multirow[b]{2}{*}{ Statistics } & \multirow[b]{2}{*}{$\mathbf{P}$} \\
\hline & Low $(n=147)$ & High $(n=282)$ & & & \\
\hline Gender, n (\%) & & & & $\chi^{2}=3.093$ & 0.079 \\
\hline Male & 64 (43.54) & $148(52.48)$ & 23 & & \\
\hline Female & $83(56.46)$ & $134(47.52)$ & 34 & & \\
\hline Age, n (\%) & & & & $\chi^{2}=37.128$ & $<0.001$ \\
\hline$<61$ & $106(72.11)$ & $116(41.13)$ & 13 & & \\
\hline$\geq 61$ & $41(27.89)$ & $166(58.87)$ & 27 & & \\
\hline $\mathrm{BMI}\left(\mathrm{x}^{-} \pm \mathrm{S}\right)$ & $22.79 \pm 2.80$ & $22.46 \pm 2.87$ & & $\mathrm{t}=1.061$ & 0.290 \\
\hline Smoking history, $\mathrm{n}(\%)$ & & & & $\chi^{2}=0.240$ & 0.624 \\
\hline Yes & $11(7.48)$ & $25(8.87)$ & 19 & & \\
\hline No & $136(92.52)$ & 257 (91.13) & 10 & & \\
\hline Drinking history, $\mathrm{n}(\%)$ & & & & $\chi^{2}=0.011$ & 0.916 \\
\hline Yes & $9(6.12)$ & $18(6.38)$ & 20 & & \\
\hline No & $138(93.88)$ & 264 (93.62) & 12 & & \\
\hline Hypertension, $\mathrm{n}(\%)$ & & & & $\chi^{2}=3.231$ & 0.072 \\
\hline Yes & $34(29.31)$ & $99(38.98)$ & 23 & & \\
\hline No & $82(70.69)$ & 155 (61.02) & 16 & & \\
\hline Diabetes, n (\%) & & & & $\chi^{2}=6.552$ & 0.010 \\
\hline Yes & $13(11.21)$ & $57(22.44)$ & 25 & & \\
\hline No & 103 (88.79) & $197(77.56)$ & 21 & & \\
\hline Hyperlipidemia, n (\%) (High HDL-C) & & & & $\chi^{2}=2.178$ & 0.140 \\
\hline Yes & $13(11.21)$ & $17(6.69)$ & 24 & & \\
\hline No & $103(88.79)$ & $237(93.31)$ & 18 & & \\
\hline Total score of admission status, $\mathrm{n}(\%)$ & & & & $\chi^{2}=7.152$ & 0.007 \\
\hline$<6$ & $100(68.03)$ & $153(54.64)$ & 24 & & \\
\hline$\geq 6$ & $47(31.97)$ & $127(45.36)$ & 17 & & \\
\hline \multicolumn{6}{|l|}{ Inpatient lab examinations } \\
\hline Leukocytes, n (\%) & & & & $Z=-0.540$ & 0.589 \\
\hline Q1 & $30(21.28)$ & $71(26.20)$ & & & \\
\hline Q2 & $40(28.37)$ & 65 (23.99) & & & \\
\hline Q3 & 35 (28.82) & $68(25.09)$ & & & \\
\hline Q4 & $36(25.53)$ & $67(24.72)$ & & & \\
\hline Neutrophils, n (\%) & & & & $Z=-0.046$ & 0.963 \\
\hline Q1 & $36(25.53)$ & $67(24.72)$ & & & \\
\hline Q2 & $34(24.11)$ & $69(25.46)$ & & & \\
\hline Q3 & $35(24.82)$ & $68(25.09)$ & & & \\
\hline Q4 & $36(25.54)$ & $67(24.73)$ & & & \\
\hline Lymphocytes, n (\%) & & & & $Z=-1.197$ & 0.231 \\
\hline Q1 & $30(21.28)$ & 73 (26.94) & & & \\
\hline Q2 & 38 (26.95) & $69(25.46)$ & & & \\
\hline Q3 & $34(24.11)$ & 65 (23.98) & & & \\
\hline Q4 & $39(27.66)$ & $64(23.62)$ & & & \\
\hline Hemoglobin, $\mathrm{n}(\%)$ & & & & $\mathrm{Z}=-0.414$ & 0.679 \\
\hline Q1 & 35 (27.78) & $51(21.16)$ & & & \\
\hline Q2 & $28(22.22)$ & $70(29.05)$ & & & \\
\hline Q3 & $31(24.60)$ & $62(25.73)$ & & & \\
\hline $\mathrm{Q} 4$ & $32(25.40)$ & $58(24.06)$ & & & \\
\hline
\end{tabular}


THE JOURNAL OF NUTRITION, HEALTH \& AGING

Table 1 (continued)

Differences in the high and low CONUT score groups

\begin{tabular}{|c|c|c|c|c|c|}
\hline \multirow[t]{2}{*}{ Variates } & \multicolumn{2}{|c|}{ Groups } & \multirow[b]{2}{*}{$\begin{array}{l}\text { Cases of adverse } \\
\text { outcomes }\end{array}$} & \multirow[b]{2}{*}{ Statistics } & \multirow[b]{2}{*}{$\mathbf{P}$} \\
\hline & Low $(n=147)$ & High $(n=282)$ & & & \\
\hline C-reactive protein, $\mathrm{n}(\%)$ & & & & $Z=-0.826$ & 0.409 \\
\hline Q1 & $40(28.37)$ & $61(22.85)$ & & & \\
\hline Q2 & $38(26.95)$ & $65(24.34)$ & & & \\
\hline Q3 & $24(17.02)$ & $78(29.21)$ & & & \\
\hline Q4 & $39(27.66)$ & $63(23.60)$ & & & \\
\hline Erythrocyte sedimentation rate, $\mathrm{n}(\%)$ & & & & $Z=-0.207$ & 0.836 \\
\hline Q1 & $10(27.78)$ & $18(23.38)$ & & & \\
\hline Q2 & $7(19.44)$ & $22(28.57)$ & & & \\
\hline Q3 & $9(25.00)$ & $19(24.47)$ & & & \\
\hline Q4 & $10(27.78)$ & $18(23.38)$ & & & \\
\hline Procalcitonin, n (\%) & & & & $Z=-1.703$ & 0.089 \\
\hline $\mathrm{Q} 1$ & $29(22.48)$ & $63(25.72)$ & & & \\
\hline Q2 & $30(23.26)$ & $66(26.94)$ & & & \\
\hline Q3 & $29(22.48)$ & $64(26.12)$ & & & \\
\hline Q4 & $41(31.78)$ & $52(21.22)$ & & & \\
\hline Urine protein, $\mathrm{n}(\%)$ & & & & $Z=-0.505$ & 0.613 \\
\hline- & $54(56.84)$ & $100(52.91)$ & & & \\
\hline+- & $22(23.16)$ & $51(26.98)$ & & & \\
\hline+ & $16(16.84)$ & $31(16.40)$ & & & \\
\hline++ & $2(2.11)$ & $3(1.59)$ & & & \\
\hline+++ & $1(1.05)$ & $4(2.12)$ & & & \\
\hline Urinary erythrocytes, $\mathrm{n}(\%)$ & & & & $\chi^{2}=1.155$ & 0.283 \\
\hline$<10.3$ & $45(45.92)$ & $102(52.58)$ & & & \\
\hline$\geq 10.3$ & $53(54.08)$ & $92(47.42)$ & & & \\
\hline Alanine aminotransferase, $\mathrm{n}(\%)$ & & & & $Z=-0.326$ & 0.744 \\
\hline Q1 & $35(24.65)$ & $65(23.90)$ & & & \\
\hline Q2 & $39(27.46)$ & $76(27.94)$ & & & \\
\hline Q3 & $29(20.42)$ & $70(25.73)$ & & & \\
\hline Q4 & $39(27.47)$ & $61(22.43)$ & & & \\
\hline Serum albumin, n (\%) & & & & $\mathrm{Z}=0.164$ & 0.870 \\
\hline Q1 & $34(24.29)$ & $64(23.97)$ & & & \\
\hline Q2 & $36(25.71)$ & $73(27.34)$ & & & \\
\hline Q3 & $38(27.14)$ & $61(22.85)$ & & & \\
\hline Q4 & $32(22.86)$ & $69(25.84)$ & & & \\
\hline Serum creatine kinase isoenzyme, $\mathrm{n}(\%)$ & & & & $Z=-0.137$ & 0.891 \\
\hline Q1 & $16(21.62)$ & $38(25.17)$ & & & \\
\hline Q2 & $29(39.19)$ & $41(27.15)$ & & & \\
\hline Q3 & $10(13.51)$ & $38(25.16)$ & & & \\
\hline Q4 & $19(25.68)$ & $34(22.52)$ & & & \\
\hline Lactate dehydrogenase, n (\%) & & & & $Z=-0.452$ & 0.651 \\
\hline Q1 & $21(29.58)$ & $33(22.76)$ & & & \\
\hline Q2 & $16(22.53)$ & $38(26.21)$ & & & \\
\hline Q3 & $15(21.13)$ & $39(26.89)$ & & & \\
\hline $\mathrm{Q} 4$ & $19(26.76)$ & $35(24.14)$ & & & \\
\hline
\end{tabular}


Table 1 (continued)

Differences in the high and low CONUT score groups

\begin{tabular}{|c|c|c|c|c|c|}
\hline \multirow[t]{2}{*}{ Variates } & \multicolumn{2}{|c|}{ Groups } & \multirow[b]{2}{*}{$\begin{array}{c}\text { Cases of adverse } \\
\text { outcomes }\end{array}$} & \multirow[b]{2}{*}{ Statistics } & \multirow[b]{2}{*}{$\mathbf{P}$} \\
\hline & Low $(n=147)$ & High $(n=282)$ & & & \\
\hline Urea nitrogen, n (\%) & & & & $Z=-0.557$ & 0.578 \\
\hline Q1 & $22(23.66)$ & $44(22.45)$ & & & \\
\hline Q2 & $25(26.88)$ & $55(28.06)$ & & & \\
\hline Q3 & $18(19.35)$ & $56(28.57)$ & & & \\
\hline Q4 & $28(30.11)$ & $41(20.92)$ & & & \\
\hline Blood creatinine, $\mathrm{n}(\%)$ & & & & $Z=-0.970$ & 0.332 \\
\hline Q1 & $36(25.35)$ & $59(22.10)$ & & & \\
\hline Q2 & $40(28.17)$ & $71(26.59)$ & & & \\
\hline Q3 & $35(24.65)$ & $71(26.59)$ & & & \\
\hline Q4 & $31(21.83)$ & $66(24.72)$ & & & \\
\hline D-D dimer, n (\%) & & & & $Z=-0.466$ & 0.641 \\
\hline Q1 & $31(23.30)$ & $61(24.11)$ & & & \\
\hline Q2 & $35(26.32)$ & $71(28.06)$ & & & \\
\hline Q3 & $32(24.06)$ & $60(23.72)$ & & & \\
\hline Q4 & $35(26.32)$ & $61(24.11)$ & & & \\
\hline
\end{tabular}

Table 2

Differences in adverse outcomes in different groups

\begin{tabular}{|c|c|c|c|c|}
\hline \multirow[t]{2}{*}{ Variates } & \multicolumn{2}{|c|}{ Groups } & \multirow[t]{2}{*}{ Statistics } & \multirow[t]{2}{*}{$\mathbf{P}$} \\
\hline & No $(n=371)$ & Yes $(n=58)$ & & \\
\hline Gender, n (\%) & & & $\chi 2=10.253$ & 0.001 \\
\hline Male & $172(46.36)$ & $40(68.97)$ & & \\
\hline Female & $199(53.64)$ & $18(31.03)$ & & \\
\hline Age, n $(\%)$ & & & $\chi 2=15.681$ & $<0.001$ \\
\hline$<61$ & $206(55.53)$ & $16(27.59)$ & & \\
\hline$\geq 61$ & $165(44.47)$ & $42(72.41)$ & & \\
\hline $\mathrm{BMI}\left(\mathrm{x}^{-} \pm \mathrm{S}\right)$ & $22.54 \pm 2.77$ & $22.82 \pm 3.39$ & $t=-0.604$ & 0.546 \\
\hline Smoking history, $\mathrm{n}(\%)$ & & & -- & 0.004 \\
\hline Yes & $25(6.74)$ & $11(18.97)$ & & \\
\hline No & $346(93.26)$ & $47(81.03)$ & & \\
\hline Drinking history, n (\%) & & & -- & 0.074 \\
\hline Yes & $20(5.39)$ & $7(12.07)$ & & \\
\hline No & $351(94.61)$ & $51(87.93)$ & & \\
\hline Hypertension, n (\%) & & & $\chi^{2}=11.240$ & 0.001 \\
\hline Yes & $104(32.60)$ & $29(56.86)$ & & \\
\hline No & $215(67.40)$ & $22(43.14)$ & & \\
\hline Diabetes, n (\%) & & & $\chi 2=0.820$ & 0.365 \\
\hline Yes & $58(18.18)$ & $12(23.53)$ & & \\
\hline No & $261(81.82)$ & $39(76.47)$ & & \\
\hline Hyperlipidemia, n (\%) & & & -- & 0.099 \\
\hline Yes & $29(9.09)$ & $1(1.96)$ & & \\
\hline No & $290(90.91)$ & $50(98.04)$ & & \\
\hline
\end{tabular}




\section{THE JOURNAL OF NUTRITION, HEALTH \& AGING}

Table 2 (continued)

Differences in adverse outcomes in different groups

\begin{tabular}{|c|c|c|c|c|}
\hline \multirow[t]{2}{*}{ Variates } & \multicolumn{2}{|c|}{ Groups } & \multirow[t]{2}{*}{ Statistics } & \multirow[t]{2}{*}{$\mathbf{P}$} \\
\hline & No $(n=371)$ & Yes $(\mathbf{n}=58)$ & & \\
\hline Total score of admission status, $\mathrm{n}(\%)$ & & & $\chi^{2}=0.011$ & 0.916 \\
\hline$<6$ & $219(59.35)$ & $34(58.62)$ & & \\
\hline$\geq 6$ & $150(40.65)$ & $24(41.38)$ & & \\
\hline \multicolumn{5}{|l|}{ Inpatient lab examinations } \\
\hline Leukocytes, n (\%) & & & $Z=-0.612$ & 0.540 \\
\hline Q1 & $86(24.16)$ & $15(26.79)$ & & \\
\hline Q2 & $96(26.96)$ & $9(16.07)$ & & \\
\hline Q3 & 87 (24.44) & $16(28.57)$ & & \\
\hline Q4 & $87(24.44)$ & $16(28.57)$ & & \\
\hline Neutrophils, n (\%) & & & $\mathrm{Z}<0.001$ & 1.000 \\
\hline $\mathrm{Q} 1$ & $87(24.44)$ & $16(28.57)$ & & \\
\hline Q2 & $92(25.84)$ & $11(19.64)$ & & \\
\hline Q3 & $89(25.00)$ & $14(25.00)$ & & \\
\hline Q4 & $88(24.72)$ & $15(26.79)$ & & \\
\hline Lymphocytes, n (\%) & & & $Z=-0.334$ & 0.738 \\
\hline Q1 & $91(25.56)$ & $12(21.43)$ & & \\
\hline Q2 & $92(25.84)$ & $15(26.79)$ & & \\
\hline Q3 & $83(23.32)$ & $16(28.57)$ & & \\
\hline Q4 & $90(25.28)$ & $13(23.21)$ & & \\
\hline Hemoglobin, n (\%) & & & $Z=-0.945$ & 0.345 \\
\hline Q1 & $72(22.93)$ & $14(26.42)$ & & \\
\hline Q2 & $89(28.34)$ & $9(16.98)$ & & \\
\hline Q3 & $81(25.80)$ & $12(22.64)$ & & \\
\hline Q4 & $72(22.93)$ & $18(33.96)$ & & \\
\hline C-reactive protein, n (\%) & & & $Z=-0.498$ & 0.619 \\
\hline Q1 & $89(25.28)$ & $12(21.43)$ & & \\
\hline Q2 & $89(25.28)$ & $14(25.00)$ & & \\
\hline Q3 & $86(24.43)$ & $16(28.57)$ & & \\
\hline Q4 & $88(25.01)$ & $14(25.00)$ & & \\
\hline Erythrocyte sedimentation rate, n (\%) & & & $Z=-0.495$ & 0.621 \\
\hline Q1 & $25(25.25)$ & $3(21.43)$ & & \\
\hline Q2 & $23(23.23)$ & $6(42.86)$ & & \\
\hline Q3 & $26(26.27)$ & $2(14.28)$ & & \\
\hline Q4 & $25(25.25)$ & $3(21.43)$ & & \\
\hline Procalcitonin, $\mathrm{n}(\%)$ & & & $Z=-0.407$ & 0.684 \\
\hline Q1 & $78(24.07)$ & $14(28.00)$ & & \\
\hline Q2 & $85(26.24)$ & $11(22.00)$ & & \\
\hline Q3 & $79(24.38)$ & $14(28.00)$ & & \\
\hline Q4 & $82(25.31)$ & $11(22.00)$ & & \\
\hline Urine protein, $\mathrm{n}(\%)$ & & & $Z=-0.540$ & 0.589 \\
\hline- & $136(54.62)$ & $18(51.43)$ & & \\
\hline+- & $64(25.70)$ & $9(25.71)$ & & \\
\hline+ & $42(16.87)$ & $5(14.29)$ & & \\
\hline++ & $4(1.61)$ & $1(2.86)$ & & \\
\hline+++ & $3(1.20)$ & $2(5.71)$ & & \\
\hline
\end{tabular}


Table 2 (continued)

Differences in adverse outcomes in different groups

\begin{tabular}{|c|c|c|c|c|}
\hline \multirow[t]{2}{*}{ Variates } & \multicolumn{2}{|c|}{ Groups } & \multirow[t]{2}{*}{ Statistics } & \multirow[t]{2}{*}{$\overline{\mathbf{P}}$} \\
\hline & No $(n=371)$ & Yes $(n=58)$ & & \\
\hline Urinary erythrocytes, $\mathrm{n}(\%)$ & & & $\chi^{2}=5.029$ & 0.025 \\
\hline$<10.3$ & $122(47.84)$ & $25(67.57)$ & & \\
\hline$\geq 10.3$ & $133(52.16)$ & $12(32.43)$ & & \\
\hline Alanine aminotransferase, $\mathrm{n}(\%)$ & & & $Z=-0.494$ & 0.621 \\
\hline Q1 & $91(25.49)$ & $9(15.79)$ & & \\
\hline Q2 & $95(26.61)$ & $20(35.09)$ & & \\
\hline Q3 & $83(23.25)$ & $16(28.07)$ & & \\
\hline Q4 & $88(24.65)$ & $12(21.05)$ & & \\
\hline Serum albumin, n (\%) & & & $Z=-0.450$ & 0.653 \\
\hline Q1 & $83(23.58)$ & $15(27.27)$ & & \\
\hline Q2 & 95 (26.99) & $14(25.45)$ & & \\
\hline Q3 & $86(24.43)$ & $13(23.64)$ & & \\
\hline Q4 & $88(25.00)$ & $13(23.64)$ & & \\
\hline Serum creatine kinase isoenzyme, $\mathrm{n}(\%)$ & & & $Z=-0.572$ & 0.568 \\
\hline Q1 & $49(24.87)$ & $5(17.86)$ & & \\
\hline Q2 & $63(31.98)$ & $7(25.00)$ & & \\
\hline Q3 & $36(18.28)$ & $12(42.86)$ & & \\
\hline Q4 & $49(24.87)$ & $4(14.28)$ & & \\
\hline Lactate dehydrogenase, $\mathrm{n}(\%)$ & & & $Z=-0.459$ & 0.646 \\
\hline Q1 & $47(24.87)$ & $7(25.93)$ & & \\
\hline Q2 & $48(25.40)$ & $6(22.22)$ & & \\
\hline Q3 & $44(23.28)$ & $10(37.04)$ & & \\
\hline Q4 & $50(26.45)$ & $4(14.81)$ & & \\
\hline Urea nitrogen, $\mathrm{n}(\%)$ & & & $Z=-0.660$ & 0.509 \\
\hline Q1 & $54(22.04)$ & $12(27.27)$ & & \\
\hline Q2 & $66(26.94)$ & $14(31.82)$ & & \\
\hline Q3 & $68(27.76)$ & $6(13.64)$ & & \\
\hline Q4 & $57(23.26)$ & $12(27.27)$ & & \\
\hline Blood creatinine, n (\%) & & & $Z=-0.465$ & 0.642 \\
\hline Q1 & $81(23.01)$ & $14(24.56)$ & & \\
\hline Q2 & 95 (26.99) & $16(28.07)$ & & \\
\hline Q3 & $91(25.85)$ & $15(26.32)$ & & \\
\hline Q4 & $85(24.15)$ & $12(21.05)$ & & \\
\hline D-D dimer, n (\%) & & & $Z=-0.144$ & 0.885 \\
\hline Q1 & $81(23.97)$ & $11(22.92)$ & & \\
\hline $\mathrm{Q} 2$ & $91(26.92)$ & $15(31.25)$ & & \\
\hline Q3 & $84(24.85)$ & $8(16.66)$ & & \\
\hline Q4 & $82(24.26)$ & $14(29.17)$ & & \\
\hline
\end{tabular}


THE JOURNAL OF NUTRITION, HEALTH \& AGING

Table 3

Multivariate regression analysis of adverse outcomes

\begin{tabular}{|c|c|c|c|c|c|c|c|}
\hline \multirow[t]{2}{*}{ Variates } & \multirow[t]{2}{*}{$\beta$} & \multirow[t]{2}{*}{ S.E } & \multirow[t]{2}{*}{ Wald } & \multirow[t]{2}{*}{$\mathbf{P}$} & \multirow[t]{2}{*}{ OR } & \multicolumn{2}{|c|}{ 95\% CI } \\
\hline & & & & & & Lower & Upper \\
\hline Constant & -2.538 & 0.490 & 26.813 & $<0.001$ & & & \\
\hline \multicolumn{8}{|l|}{ Gender } \\
\hline Male & Ref & & & & & & \\
\hline Female & -1.093 & 0.435 & 6.312 & 0.012 & 0.335 & 0.143 & 0.786 \\
\hline \multicolumn{8}{|l|}{ Age } \\
\hline$<61$ & Ref & & & & & & \\
\hline$\geq 61$ & 1.492 & 0.496 & 9.065 & 0.003 & 4.447 & 1.683 & 11.747 \\
\hline \multicolumn{8}{|c|}{ Hypertension } \\
\hline No & Ref & & & & & & \\
\hline Yes & 0.974 & 0.421 & 5.352 & 0.021 & 2.648 & 1.160 & 6.042 \\
\hline \multicolumn{8}{|c|}{ Urinary erythrocytes } \\
\hline$<10.3$ & Ref & & & & & & \\
\hline$\geq 10.3$ & -0.959 & 0.429 & 4.997 & 0.025 & 0.383 & 0.165 & 0.888 \\
\hline
\end{tabular}

To analyze the predictive effects of CONUT score on adverse outcomes, we assessed the difference in baseline data. The CONUT score $\leq 4$ was set as the low CONUT score group, while the CONUT score $\geq 5$ was set as the high CONUT score group. Significant differences were observed in three groups of patients in age, diabetes and total score of admission status (Table 1) . Grouped by whether there was any adverse outcome, significant differences were observed in gender, age, smoking history, hypertension and urine red blood cells (Table 2) . After adjusting the influences of many confounding factors, multivariate logistic regression analysis of adverse outcomes was applied and results showed that gender, age, hypertension, urinary red blood cells and CONUT score were the risk factors for adverse outcomes (Table 3). Results of Table 4 showed that the risk of adverse outcomes in female patients was 0.332 times that of males, which might be related to hormone levels. The risk of adverse outcomes in older patients was 4.054 times that of younger patients $(\mathrm{OR}=4.054,95 \% \mathrm{CI}$ : 1.483 11.080). Furthermore, the risk of adverse outcomes in patients with hypertension was 2.900 times that of patients without hypertension $(\mathrm{OR}=2.900,95 \%$ CI: $1.246-6.751)$. The risk of having adverse outcomes in the high CONUT score group was 3.598 times higher than that in the low CONUT score group (OR $=3.598,95 \%$ CI: $1.003-12.902)$.

Among patients $\geq 61$ years old, the risk of adverse outcomes in the high CONUT score group was 6.191 times that of the low CONUT score group (OR $=6.191,95 \%$ CI: 1.431 26.785). However, among patients younger than 61 years old, no statistical difference was observed in the risk of adverse outcomes between the high and the low CONUT groups (P $>0.05)$. Among the non-diabetic patients, the risk of adverse outcomes in the high CONUT group was 11.678 times that of the low CONUT group $(\mathrm{OR}=11.678,95 \%$ CI: 2.754-49.41). While among the diabetic patients, no statistical difference was observed in the risk of adverse outcomes between the high and the low CONUT groups $(\mathrm{P}>0.05)$. For the patients who had a total score of admission status $<6$, the risk of adverse outcomes in the high CONUT score group was 8.216 times that of the low CONUT score group (OR $=8.216,95 \%$ CI: 2.439-27.682). However, For the patients who had a total score of admission status $\geq 6$, no statistical difference was observed in the risk of adverse outcomes between the high and the low CONUT groups $(\mathrm{P}>0.05)$.

In conclusion, the nutritional status of COVID-19 patients is closely related to clinical outcomes. High CONUT score, male, combined with hypertension or diabetes or advanced age are the risk factors, and patients with these factors have a higher risk of adverse outcomes.

Conflict of interest: The authors declare that they have no conflict of interest.

Funding source: The study was approved by the Hubei Provincial Hospital of Traditional Chinese Medicine (2020YFC0841600).

Ethical approval: The study was approved by the ethics committee of Hubei Provincial Hospital of Traditional Chinese Medicine (HBZY2020-C14-01).

Acknowledgement: We thank all the participants who were involved in this study and Hubei Provincial Hospital of Traditional Chinese Medicine for providing assistance.

\section{References}

1. Zou X, Chen K, Zou J, Han P, Hao J, Han Z. Single-cell RNA-seq data analysis on the receptor ACE2 expression reveals the potential risk of different human organs vulnerable to 2019-nCoV infection. Frontiers of medicine. 2020;14(2):185-92. doi: 10.1007/s11684-020-0754-0.

2. Zhao Y, Zhao Z, Wang Y, Zhou Y, Ma Y, Zuo W. Single-cell RNA expression profiling of ACE2, the putative receptor of Wuhan 2019-nCov. bioRxiv. 2020-2020 Preprint available at https://www.biorxiv.org/content/10.1101/2020.01.26.919985v1.

3. Zhang H, Kang Z, Gong H, Xu D, Wang J, Li Z, et al. The digestive system is a potential route of 2019-nCov infection: a bioinformatics analysis based on singlecell transcriptomes. bioRxiv. 2020.Preprint available at https://www.biorxiv.org/ content/10.1101/2020.01.30.927806v1.

4. Xu H, Zhong L, Deng J, Peng J, Dan H, Zeng X, et al. High expression of ACE2 receptor of 2019-nCoV on the epithelial cells of oral mucosa. International journal of 
Table 4

Analysis of the influences of CONUT score on adverse outcomes

\begin{tabular}{|c|c|c|c|c|c|c|c|}
\hline \multirow[t]{2}{*}{ Variates } & \multirow[t]{2}{*}{$\beta$} & \multirow[t]{2}{*}{ S.E } & \multirow[t]{2}{*}{ Wald } & \multirow[t]{2}{*}{$\mathbf{P}$} & \multirow[t]{2}{*}{ OR } & \multicolumn{2}{|c|}{ 95\% CI } \\
\hline & & & & & & Lower & Upper \\
\hline Constant & -3.409 & 0.736 & 21.435 & $<0.001$ & & & \\
\hline \multicolumn{8}{|l|}{ Gender } \\
\hline Male & Ref & & & & & & \\
\hline Female & -1.102 & 0.442 & 6.227 & 0.013 & 0.332 & 0.140 & 0.789 \\
\hline \multicolumn{8}{|l|}{ Age } \\
\hline$<61$ & Ref & & & & & & \\
\hline$\geq 61$ & 1.400 & 0.513 & 7.446 & 0.006 & 4.054 & 1.483 & 11.080 \\
\hline \multicolumn{8}{|c|}{ Hypertension } \\
\hline No & Ref & & & & & & \\
\hline Yes & 1.065 & 0.431 & 6.104 & 0.013 & 2.900 & 1.246 & 6.751 \\
\hline \multicolumn{8}{|l|}{ Diabetes } \\
\hline No & Ref & & & & & & \\
\hline Yes & -0.589 & 0.511 & 1.328 & 0.249 & 0.555 & 0.204 & 1.511 \\
\hline \multicolumn{8}{|c|}{ Nutritional treatment } \\
\hline No & Ref & & & & & & \\
\hline Yes & 1.363 & 0.618 & 1.437 & 0.265 & 0.517 & 0.271 & 2.318 \\
\hline \multicolumn{8}{|c|}{ Total score of admission status } \\
\hline$<6$ & Ref & & & & & & \\
\hline$\geq 6$ & 0.190 & 0.420 & 0.203 & 0.652 & 1.209 & 0.529 & 2.760 \\
\hline \multicolumn{8}{|c|}{ Blood protein level } \\
\hline Low & Ref & & & & & & \\
\hline High & 0.316 & 0.518 & 0.327 & 0.517 & 1.328 & 0.472 & 3.762 \\
\hline \multicolumn{8}{|c|}{ Urinary erythrocytes } \\
\hline$<10.3$ & Ref & & & & & & \\
\hline$\geq 10.3$ & -1.014 & 0.446 & 5.173 & 0.023 & 0.363 & 0.151 & 0.869 \\
\hline \multicolumn{8}{|l|}{ I index } \\
\hline$<20$ & Ref & & & & & & \\
\hline $20-24$ & 1.025 & 0.125 & 0.118 & 0.011 & 0.382 & 0.206 & 1.346 \\
\hline$<24$ & Ref & & & & & & \\
\hline \multicolumn{8}{|c|}{ CONUT score } \\
\hline Low & Ref & & & & & & \\
\hline High & 1.280 & 0.652 & 3.861 & 0.049 & 3.598 & 1.003 & 12.902 \\
\hline
\end{tabular}

Table 5

Analysis of the influences of CONUT score on adverse outcomes in different subgroups

\begin{tabular}{|c|c|c|c|c|}
\hline \multirow[t]{2}{*}{ Variates } & \multicolumn{2}{|l|}{ Groups (CONUT score) } & \multirow[t]{2}{*}{$\mathbf{P}$} & \multirow[t]{2}{*}{ OR $(95 \% \mathrm{CI})$} \\
\hline & $\begin{array}{c}\text { Low (adverse outcomes/number of } \\
\text { patients) }\end{array}$ & $\begin{array}{c}\text { High (adverse outcomes/number of } \\
\text { patients) }\end{array}$ & & \\
\hline \multicolumn{5}{|c|}{ Age, $\mathrm{n}(\%)$} \\
\hline$<61$ & $4 / 106$ & $12 / 116$ & 0.589 & $2.942(0.919-9.424)$ \\
\hline$\geq 61$ & $2 / 41$ & $40 / 166$ & 0.003 & $6.191(1.431-26.785)$ \\
\hline \multicolumn{5}{|c|}{$\mathrm{DM}, \mathrm{n}(\%)$} \\
\hline Yes & $2 / 13$ & $10 / 57$ & 1.000 & $1.170(0.224-6.118)$ \\
\hline No & $2 / 103$ & $37 / 197$ & $<0.001$ & $11.678(2.754-49.512)$ \\
\hline \multicolumn{5}{|c|}{ Total score, n (\%) } \\
\hline$<4$ & $3 / 100$ & $31 / 153$ & $<0.001$ & $8.216(2.439-27.682)$ \\
\hline$\geq 4$ & $3 / 47$ & $21 / 127$ & 0.064 & $2.906(0.824-10.241)$ \\
\hline
\end{tabular}




\section{THE JOURNAL OF NUTRITION, HEALTH \& AGING}

oral science $2020 ; 12(1): 8$ doi: $10.1038 /$ s41368-020-0074-x.

5. Yang Y, Peng F, Wang R, Yang M, Guan K, Jiang T, et al. Corrigendum to "The deadly coronaviruses: The 2003 SARS pandemic and the 2020 novel coronavirus epidemic in China" [J. Autoimmun. 109C (2020) 102434]. Journal of autoimmunity. 2020;111:102487. doi: 10.1016/j.jaut.2020.102487.

6. Lillie PJ, Samson A, Li A, Adams K, Capstick R, Barlow GD, et al. Nove coronavirus disease (Covid-19): The first two patients in the UK with person to person transmission. The Journal of infection. 2020;80(5):578-606. doi: 10.1016/j. jinf.2020.02.020

7. Spina S, Marrazzo F, Migliari M, Stucchi R, Sforza A, Fumagalli R. The response of Milan's Emergency Medical System to the COVID-19 outbreak in Italy. Lancet (London, England). 2020;395(10227):e49-e50. doi: 10.1016/s0140-6736(20)30493-1.

8. Cengiz O, Kocer B, Sürmeli S, Santicky MJ, Soran A. Are pretreatment serum albumin and cholesterol levels prognostic tools in patients with colorectal carcinoma? Medical science monitor : international medical journal of experimental and clinical research. 2006;12(6):Cr240-7.

9. de Ulíbarri Pérez JI, Fernández G, Rodríguez Salvanés F, Díaz López AM. Nutritional screening; control of clinical undernutrition with analytical parameters. Nutricion hospitalaria. 2014;29(4):797-811. doi: 10.3305/nh.2014.29.4.7275.
10. Mohri Y, Inoue Y, Tanaka K, Hiro J, Uchida K, Kusunoki M. Prognostic nutritional index predicts postoperative outcome in colorectal cancer. World journal of surgery. 2013;37(11):2688-92. doi: 10.1007/s00268-013-2156-9.

11. Honda H, Qureshi AR, Heimbürger O, Barany P, Wang K, Pecoits-Filho R, et al. Serum albumin, C-reactive protein, interleukin 6 , and fetuin a as predictors of malnutrition, cardiovascular disease, and mortality in patients with ESRD. American journal of kidney diseases : the official journal of the National Kidney Foundation. 2006;47(1):139-48. doi: 10.1053/j.ajkd.2005.09.014.

12. Kang R, Li P, Wang T, Li X, Wei Z, Zhang Z, et al. Apolipoprotein E epsilon 2 allele and low serum cholesterol as risk factors for gastric cancer in a Chinese Han population. Scientific reports. 2016;6:19930. doi: 10.1038/srep19930.

13. Vicente Conesa MA, Garcia-Martinez E, Gonzalez Billalabeitia E, Chaves Benito A, Garcia Garcia T, Vicente Garcia V, et al. Predictive value of peripheral blood lymphocyte count in breast cancer patients treated with primary chemotherapy. Breas (Edinburgh, Scotland). 2012;21(4):468-74. doi: 10.1016/j.breast.2011.11.002.

14. Hoskin PJ, Rojas AM, Peiris SN, Mullassery V, Chong IY. Pre-treatment haemoglobin and peripheral blood lymphocyte count as independent predictors of outcome in carcinoma of cervix. Clinical oncology (Royal College of Radiologists (Great Britain)). 2014;26(4):179-84. doi: 10.1016/j.clon.2013.11.023 